Please do not remove this page

RMIT

UNIVERSITY

\title{
Multiple channel vector sum phase shifter using acousto-optic WDM
}

Bui, Lam; Mitchell, Arnan; Ghorbani, Kamran

https://researchrepository.rmit.edu.au/esploro/outputs/9921859664101341/filesAndLinks?institution=61RMIT_INST\&index=null

Bui, L., Mitchell, A., \& Ghorbani, K. (2005). Multiple channel vector sum phase shifter using acousto-optic WDM. Proceedings of the IEEE International Topical Meeting on Microwave Photonics, 1-4.

https://researchrepository.rmit.edu.au/discovery/fulldisplay/alma9921859664101341/61RMIT_INST:Resea rchRepository

Repository homepage: https://researchrepository.rmit.edu.au

(c) 2005 IEEE. Personal use of this material is permitted. However, permission to reprint/republish this material for advertising or promotional purposes or for creating new collective works for resale or redistribution to servers or lists, or to reuse any copyrighted component of this work in other works must be obtained from the IEEE.

Downloaded On 2023/04/26 22:58:09 +1000 


\title{
Multiple channel Vector Sum Phase Shifter Using Acousto-optic WDM
}

\author{
Lam Anh Bui, Arnan Mitchell and Kamran Ghorbani \\ SECE, RMIT University, GPO Box 2476V, Melbourne 3001, Australia.
}

\begin{abstract}
This paper proposes a novel technique to extend the capability of the vector sum phase shifter from a single channel to multiple channels for phased array antenna applications. Each channel is implemented using a distinct optical wavelength. Selective control of each channel is performed using an acousto-optic polarisation coupler. The concept is successfully demonstrated for two individually controlled channels. For each channel, a continuously variable frequency-linear phase shift is demonstrated between $\mathrm{DC}$ and $7 \mathrm{GHz}$ with the phasing range exceeding $100^{\circ}$. It is anticipated that up to 10 channels could be simultaneously operated in a single device.
\end{abstract}

Index Terms - Microwave phase shifter, microwave antenna arrays, optical fiber delay lines, optical fiber polarization, phase shifter, phased array radar, surface acoustic wave devices.

\section{INTRODUCTION}

Modern electronic warfare requires antenna systems that can perform multiple functions and operate over a large frequency band [1]. A major framework that enables these capabilities is phased array antenna (PAA) technology [2]. Many investigations in the past several years have attempted to realise broadband, electronically scanned PAAs [3]. It has been established that photonics is an attractive technology for this purpose due to its broad bandwidth, immunity to EMI, light weight and flexible system implementation [4]. A drawback of this technology is the high cost of both component and implementation.

Recently, we have demonstrated the vector sum phase shifter (VSPS) as an alternative to the true time delay solution for PAAs. A detailed description of the vector sum phase shifter (VSPS) is presented elsewhere [5]. A brief explanation of the VSPS is given here for the reader's convenience. A schematic of the VSPS is presented in Fig. 1. The RF signal to be phase shifted is split into two components with a ratio of $x:(1-x)$. Each component is transmitted through a delay line of different length. The differentially delayed components are then summed using an in-phase combiner. Continuous phase control is achieved by varying the splitting ratio $x$.

Several photonic realisations of the VSPS have been demonstrated. They include utilisation of a variable directional coupler $[5,6]$ and of polarisation mode

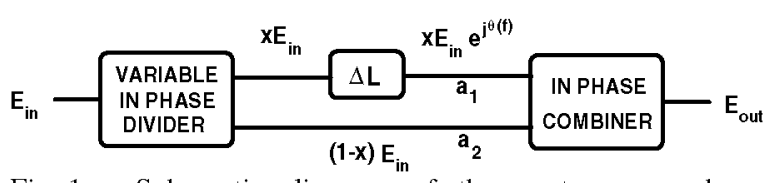

Fig. 1. Schematic diagram of the vector sum phase shifter

dispersion in a high birefringence (HiBi) fibre $[6,7]$. Among these implementations, utilisation of polarisation mode dispersion in a HiBi fibre is the most elegant solution. This device consists of an electronicallycontrolled acousto-optic polarisation coupler (AOPC) and a length of HiBi fibre. Single channel operation of this device has been demonstrated [7]. It is advantageous to extend the operation of the device to multiple channels. Conceiving such a technique is thus worth investigating.

In this paper, we demonstrate a novel VSPS device whose channels can simultaneously be utilised and independently tuned. This paper is arranged as follows. In Section II, the AOPC principle of operation is briefly explained. The AOPC is characterised and multiwavelength operation of the device is demonstrated. In Section III, the two-channel VSPS is measured and continuously variable and simultaneously tuned channels are demonstrated. In Section IV, the practicality and future improvements of the device are discussed. The concluding remarks are given in Section $\mathrm{V}$.

\section{ACOUSTO-OPTIC POLARISATION COUPLER}

\section{A. Principle of Operation}

The acousto-optic polarisation coupler (AOPC) employed for this VSPS is similar to the device reported in [8]. A schematic diagram of this device is presented in Fig. 2. This device consists of an optical waveguide loaded by a thin-film acoustic waveguide. The optical waveguide was realised by in-diffusion of Titanium into $\mathrm{X}$-cut Y-propagating $\mathrm{LiNbO}_{3}$. The diffusion conditions were customised so that the waveguide supports both TE and TM modes with similar propagation loss. The acoustic waveguide is realised as a thin film of $\mathrm{SiO}_{2}$ [8]. 


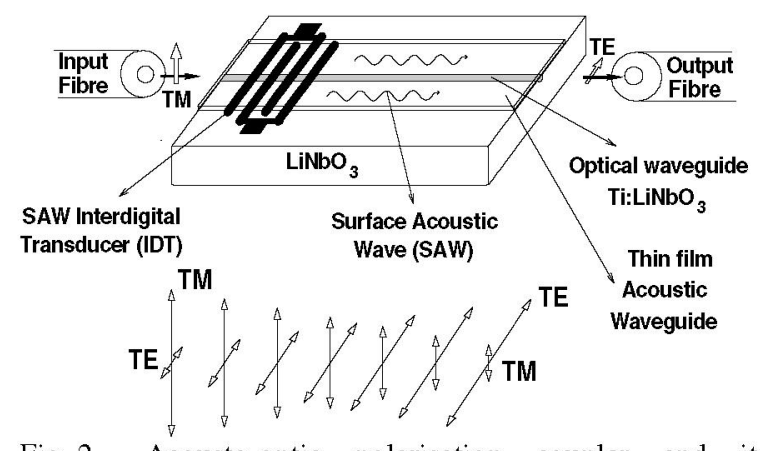

Fig. 2. Acousto-optic polarisation coupler and its principle of operation.

The acoustic waveguide makes a small angle with respect to the optical waveguide so that the interaction between the acoustic wave and the optical wave is apodised along the length of the device. This allows windowing of the coupling strength between the two orthogonal modes which is implemented to reduce the side lobe level of the wavelength selective characteristic of the AOPC.

The principle of operation of the AOPC is illustrated in Fig. 2. When an acoustic wave is set up in the device, it creates a periodic mechanical stress on top of the optical waveguide. This stress periodically perturbs the material dielectric tensor and hence couples the power between the two polarisation modes for optical wavelengths that satisfy the phase matching condition. The strength of this coupling could then be varied by changing the power of the acoustic wave. The AOPC is therefore effectively an electronically variable polarisation coupler.

\section{B. Device Characterisation}

The device of [8] was reproduced and packaged at the Microelectronics Materials Technology Centre (MMTC) within RMIT University. The guiding of both polarisations was characterised, in which TE mode has $1.5 \mathrm{~dB}$ lower insertion loss than TM mode. The coupling characteristic of the device was also measured at several optical wavelengths. The acoustic frequency that satisfies the phase-matching condition at each wavelength was experimentally determined. It is found that the acoustic frequencies that satisfy the phase matching condition for the wavelengths of $1548.13 \mathrm{~nm}$ and $1551.73 \mathrm{~nm}$ are 172.1 and $171.68 \mathrm{MHz}$ respectively. Light was launched into the device in TM mode using polarisation maintaining fibre. Output power in each polarisation was analysed using a polarisation beam splitter (PBS). Fig. 3 presents the coupling characteristic versus the driving acoustic power measured at $1550 \mathrm{~nm}$.

It is evident from Fig. 3 that the output polarisation remains the same as the input polarisation (TM) at low acoustic power. As the driving power increases, the power in TM mode slowly transfers into TE mode and at $15 \mathrm{dBm}$, most of the power has been converted to TE. The achieved extinction ratio at maximum conversion is around $10 \mathrm{~dB}$. It is thus possible to vary the ratio of

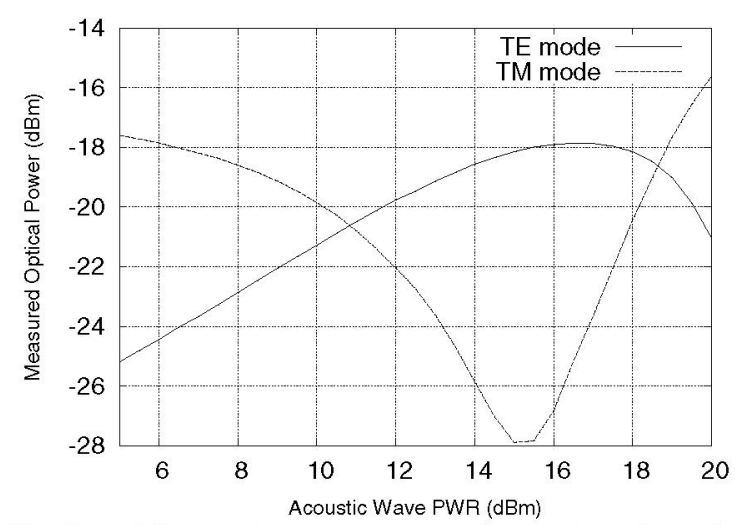

Fig. 3. Measured output power in each polarisation versus driving acoustic power at $1550 \mathrm{~nm}$.

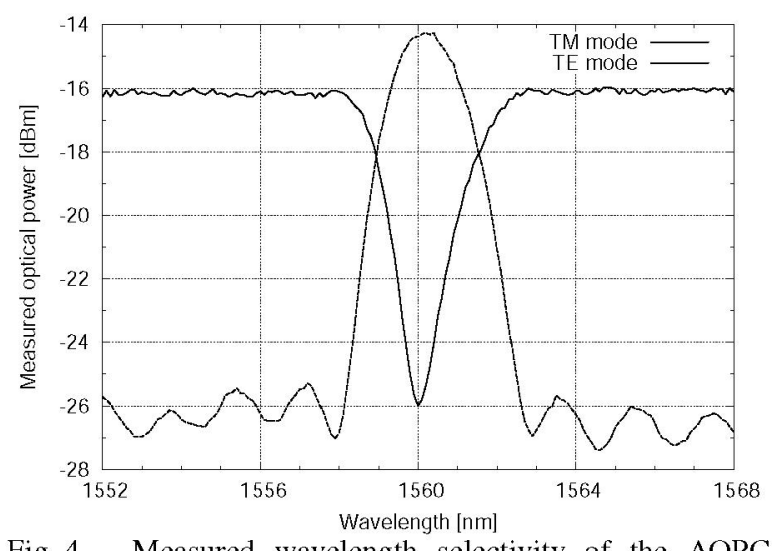

Fig. 4. Measured wavelength selectivity of the AOPC driven by a $172.91 \mathrm{MHz}$ acoustic wave with power level of $13 \mathrm{dBm}$.

power between output TE and TM within the range from 0 to $-10 \mathrm{~dB}$.

The wavelength selective coupling of the AOPC is also measured under a constant driving acoustic power of 13 $\mathrm{dBm}$. The measured power in each polarisation versus the optical wavelength for an acoustic frequency that has been phase-matched to $1560 \mathrm{~nm}$ is presented in Fig. 4. It is evident that the device exhibits a band-pass response. The pass-band bandwidth approximates $2 \mathrm{~nm}$. It is thus concluded that the channel separation for a VSPS that employed this AOPC should be larger than $2 \mathrm{~nm}$ to minimise wavelength cross-talk.

\section{DEMONSTRATION OF MULTI-CHANNEL VSPS}

\section{A. Experimental Setup}

A multi-channel VSPS is an extension of the single channel VSPS presented in [7]. Fig. 5 presents the demonstration setup. The system is demonstrated for two independent channels. Each channel consists of a separate laser; an optical Mach Zehnder Modulator (MZM); several shared optical components (as shown in Fig. 5) and a photo detector.

For this demonstration, the two selected wavelengths are $\lambda_{1}=1548.13 \mathrm{~nm}$ and $\lambda_{2}=1551.73 \mathrm{~nm}$. After 


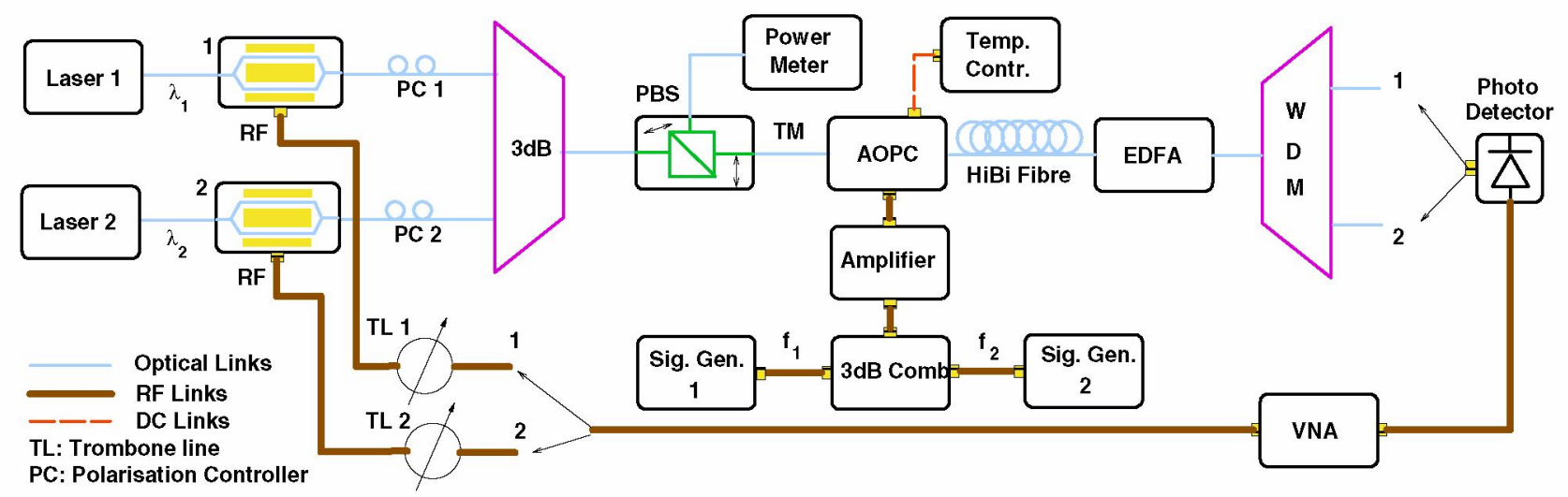

Fig. 5. Experimental setun to demonstrate dual channel VSPS
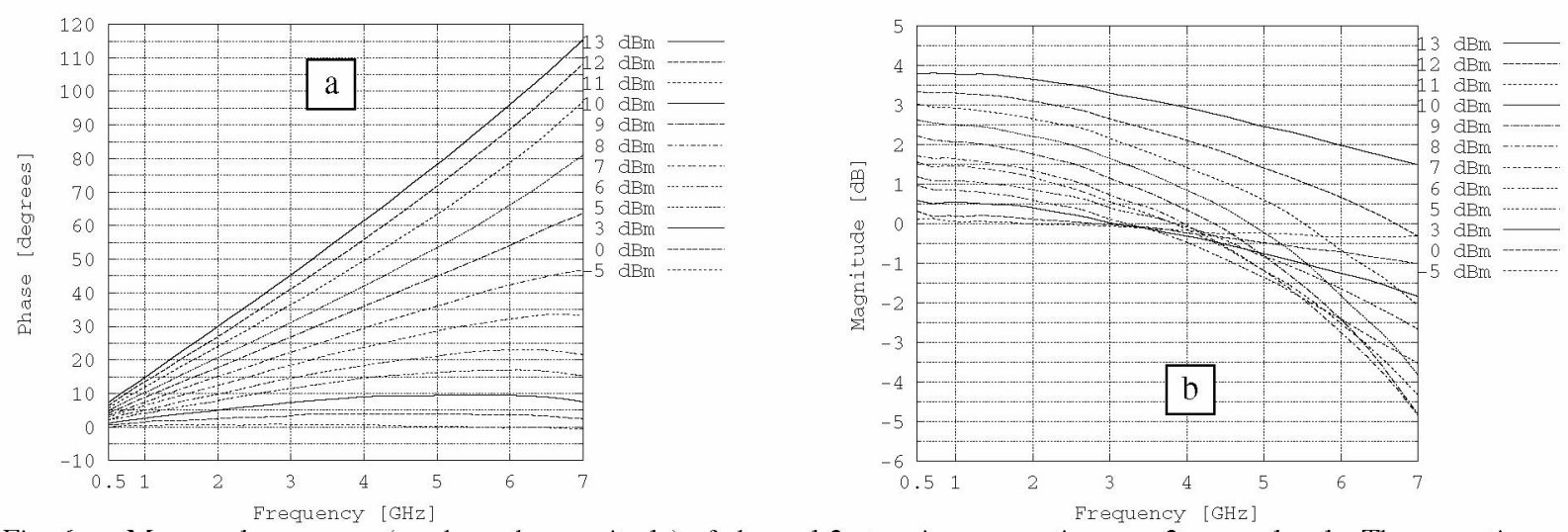

Fig. 6. Measured responses (a- phase; b-magnitude) of channel 2 at various acoustic wave 2 power levels. The acoustic wave 1 is not launched.

modulation, the two wavelengths with their sidebands are combined using a $3 \mathrm{~dB}$ coupler. The polarisation controllers PC 1 and PC2 are employed to align $\lambda_{1}$ and $\lambda_{2}$ to one of the major polarisation axes of the AOPC (TM in this case). This is achieved with the help of a polarisation beam splitter (PBS) as shown in Fig. 5.

Polarisation coupling of both $\lambda_{1}$ and $\lambda_{2}$ are then individually controlled by the AOPC using two acoustic waves whose frequencies are $\mathrm{f}_{1}=172.1 \mathrm{MHz}$ and $\mathrm{f}_{2}=171.68 \mathrm{MHz}$ respectively. The acoustic waves are generated by combining the frequency components provided by Sig. Gen. 1 and Sig. Gen. 2 through a $3 \mathrm{~dB}$ coupler. At the AOPC output, each wavelength component generally consists of both TE and TM polarisations. After traveling through a length of $\mathrm{HiBi}$ fibre, a time lag is incurred between the two orthogonal polarisations. It is assumed that for the first order approximation within the 1530-1560 $\mathrm{nm}$ band, the time lag is wavelength independent so that they are the same for both $\lambda_{1}$ and $\lambda_{2}$. Both wavelengths are amplified using an Erbium Doped Fibre Amplifier (EDFA), and then separated by a WDM demultiplexer. Finally, each wavelength is detected by a photo detector. The photo detector sums the differentially delayed components (TE and TM components) and thus performs the vector sum phasing scheme.

With the absence of the acoustic waves, the electrical length of channel 1 is normalized to that of channel 2 to permit comparison. This is achieved by using RF phasematched cables together with variable microwave trombone lines (TL1 and TL2) as shown in Fig. 5.

\section{B. Experimental Results}

Initially, both the signal generators (Sig. Gen $1 \& 2$ ) are turned off and the laser outputs are adjusted to $1 \mathrm{~mW}$. The vector network analyzer (VNA) is configured to measure channel 2 and is calibrated to the transmission response of this channel. Sig. Gen. 2 (phase-matched to $\lambda_{2}$ ) is turned on and channel 2 response for several discrete values of acoustic wave 2 power (a control variable for channel 2) is measured. Fig. 6 presents these measured results.

The inter-dependence of channels 1 and 2 are investigated by measuring several system scenarios. Particularly, the same measurements are performed on channel 2 however this time the $13 \mathrm{dBm}$ acoustic wave 1 is launched together with the acoustic wave 2 . The roles of channel 1 and channel 2 are then swapped. Channel 1 is measured with the absence and then with the presence of the acoustic wave 2. It is found that similar responses (which should ideally be identical) are obtained for the four measurement scenarios. The phase shifts at $7 \mathrm{GHz}$ for each of these scenarios are presented in Fig. 7. 


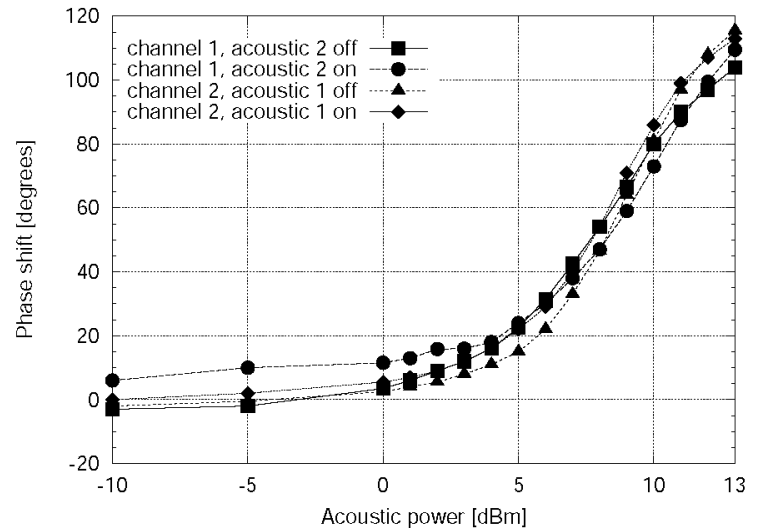

Fig. 7. Measured phase shift at $7 \mathrm{GHz}$ versus the driving acoustic power for the four considered scenarios.

\section{DISCUSSION}

It is evident from Fig. 7 that the phase responses of the four scenarios are very similar. It is thus concluded that channels 1 and 2 could be tuned simultaneously and individually. The discrepancies among the phase responses indicate coupling between channels. This coupling could be significantly reduced by improving the wavelength selectivity of the AOPC or by increasing the separation between the wavelength channels.

There are several factors that limit the total number of channels. One of them is the channel coupling discussed above. Another one is the EDFA bandwidth. For the channel separation of $3 \mathrm{~nm}$, the EDFA bandwidth of $1530-1560 \mathrm{~nm}$ could easily fit 10 channels in a single VSPS device. With the increasing number of channels, the acoustic power also increases accordingly. It is anticipated that the ultimate number of channels would be limited by the threshold acoustic power at which the AOPC becomes unstable due to the thermal effects. Issues associated with acoustic wave amplifications, heat dissipation and temperature control become significant for a large number of channels and need to be addressed to make the device practical.

It is anticipated that the multi-channel VSPS is suitable for phased array antenna applications. Employing this technology permits one device to serve several array elements, hence reducing the number of components required for the beam forming network. Investigations are currently underway to integrate this device into a practical phased array antenna demonstrator.

In the above demonstration, the electrical lengths between channels 1 and 2 are required to be matched. This is only necessary when implementing the system using discrete components. It is proposed that if the MZMs and WDM multiplexers and de-multiplexers are to be integrated, the lengths among various parts could be easily equalized. Finally the spread in amplitude responses in Fig. 6-b is due to $1.5 \mathrm{~dB}$ difference in loss between TE and TM as evident in Fig. 4. This problem is not intrinsic to the VSPS and could be reduced by improving the waveguide fabrication for the AOPC.

\section{CONCLUSIONS}

We have proposed a novel technique to extend the capability of the VSPS from a single channel to multiple channels. This technique has been successfully demonstrated for two simultaneously operated and individually controlled channels. It is anticipated that up to 10 channels could be simultaneously operated in a single device. For a large number of channels, the issues associated with acoustic power, heat dissipation and temperature control need to be properly addressed for system stability.

\section{ACKNOWLEDGEMENT}

The authors wish to acknowledge the technical assistances of Yuxun Cao, David Welsh and the financial support of DSO National Laboratories and RMIT University.

\section{REFERENCES}

[1] J. C. Veihl, R. E. Hodges, D. McGrath, and C. Monzon, "Reconfigurable aperture decade bandwidth array," presented at Antennas and Propagation Society International Symposium, 2000. IEEE, 2000.

[2] R. A. Axford, Jr., D. R. Major, and J. W. Rockway, "An assessment of multi-function phased array antennas for modern military platforms," presented at Phased Array Systems and Technology, 2003. IEEE International Symposium on, 2003.

[3] A. N. Riza and J. B. Thompson, Selected papers on photonic control systems for phased array antennas: SPIE Optical Engineering Press, 1997.

[4] P. J. Matthews, "Practical photonic beamforming," presented at Microwave Photonics, 1999. MWP 99. International Topical Meeting on, 1999.

[5] L. A. Bui, A. Mitchell, K. Ghorbani, and T.-H. Chio, "Wideband RF photonic vector sum phase-shifter," Electronics Letters, vol. 39, pp. 536-537, 2003.

[6] L. A. Bui, A. Mitchell, and T.-H. Chio, "Wideband Vector Sum Phase Shifter With Minimal Coherent Interference," presented at COIN 2003 Conference incorporating ACOFT, Melbourne, Australia, 2003.

[7] L. A. Bui, A. Mitchell, K. Ghorbani, and T.-H. Chio, "Electronically tunable vector sum phase shifter using acousto-optic polarisation coupler," submitted to the 30th Australian Conference on Optical Fibre Technology (ACOFT), Sydney, Australia, 2005.

[8] H. Mendis, A. Mitchell, I. Belski, M. Austin, and O. A. Peverini, "Design, realisation and analysis of an apodised, film-loaded acousto-optic tunable filter," Applied Physics B: Lasers and Optics, vol. 73, pp. 489-493, 2001. 